\title{
Functional hemispherectomy: can preoperative imaging predict outcome?
}

\author{
Alexander G. Weil, MD, FRCSC, ${ }^{1}$ Aria Fallah, MD, MSc, FRCSC, ${ }^{2}$ Shelly Wang, MD, MPH, FRCSC, ${ }^{3}$ \\ George M. Ibrahim, MD, PhD, FRCSC, ${ }^{4}$ Lior M. Elkaim, MD, ${ }^{5}$ Prasanna Jayakar, MD, PhD, ${ }^{3}$ \\ Ian Miller, MD, ${ }^{3}$ Sanjiv Bhatia, MD, ${ }^{3}$ Toba N. Niazi, MD, ${ }^{3}$ and John Ragheb, MD ${ }^{3}$ \\ 'Division of Neurosurgery, Sainte Justine Hospital, Montreal, Quebec, Canada; ${ }^{2}$ Department of Neurosurgery, UCLA Mattel \\ Children's Hospital, David Geffen School of Medicine at University of California, Los Angeles, California; ${ }^{3}$ Division of Pediatric \\ Neurosurgery, Department of Neurosurgery, Brain Institute, Miami Children's Hospital, Miami, Florida; ${ }^{2}$ Division of Neurosurgery, \\ The Hospital for Sick Children, The Hospital for Sick Children Research Institute, Toronto, Ontario, Canada; and ${ }^{5}$ Department of \\ Neurosurgery, McGill University, Montreal, Quebec, Canada
}

OBJECTIVE Although hemispherectomy is an effective treatment for children with intractable hemispheric epilepsy syndromes, as many as $40 \%$ of patients eventually develop seizure recurrence. The causes of seizure recurrence in these patients are incompletely understood. The authors sought to evaluate the efficacy of hemispherectomy at their center and determine whether contralateral MRI abnormalities can predict seizure recurrence.

METHODS A retrospective review of consecutive hemispherectomies performed at Miami Children's Hospital between January 2000 and June 2014 was performed. Time-to-event analysis was performed. The "event" was defined as any seizures following resective epilepsy surgery (not including seizures in the first postoperative week and auras). Several preoperative variables were analyzed to determine their suitability to predict seizure recurrence following surgery.

RESULTS Sixty-nine patients (44 boys) with a mean age of $8.2 \pm 5.9$ years (range 0.1-20.8 years) underwent 72 hemispherectomies; 67 of these were functional hemispherectomies, while another 5 were completion of a previous functional hemispherectomy (2 completions of functional hemispherectomies, 3 anatomical hemispherectomies). The duration of epilepsy was $5.8 \pm 5.5$ years with 66 cases $(91.7 \%)$ having daily seizures. Etiology included stroke $(n=28)$, malformation of cortical development $(n=11)$, hemimegalencephaly $(n=11)$, encephalitis $(n=13)$, and other $(n=7)$. Engel class I outcome was achieved in $59(86 \%)$ and 56 (81\%) patients at 1 and 2 years of follow-up, respectively. The mean time to seizure recurrence was $33.5 \pm 31.1$ months. In univariate analyses, the absence of contralateral abnormalities on MRI (HR 4.09, 95\% Cl 1.41-11.89, $p=0.009$ ) was associated with a longer duration of seizure freedom. The presence of contralateral MRI abnormalities was associated with contralateral ictal seizures on preoperative scalp EEG $(p=0.002)$. Fifteen patients experienced 20 complications (20/72, 27.8\%), including the development of hydrocephalus necessitating CSF diversion in 9 cases (13\%), hygroma in 1, hemispheric edema in 1, aseptic meningitis in 2, postoperative hemorrhage in 2, infection in 2, ischemic stroke in 2, and blood transfusion-contracted hepatitis $\mathrm{C}$ in 1 case.

CONCLUSIONS Patients with bihemispheric abnormalities, as evidenced by contralateral MRI abnormalities, have a higher risk of earlier seizure recurrence following functional hemispherectomy.

https://thejns.org/doi/abs/10.3171/2019.12.PEDS19370

KEYWORDS hemispherectomy; MRI; EEG; epilepsy; medically refractory; prognostic factors

A LTHOUGH hemispherectomy is an effective treatment for children with intractable hemispheric epilepsy syndromes, up to $40 \%$ of patients go on to develop seizure recurrence. ${ }^{22}$ The causes of seizure recurrence in these patients are incompletely understood.
Traditionally, the ideal candidates for hemispherectomy have concordant unilateral findings on neuroimaging and electrophysiological studies. ${ }^{12,16,19,22,24,26,27}$ However, whether the presence of bilateral preoperative MRI abnormalities is truly associated with a worse seizure outcome fol-

ABBREVIATIONS EVD = external ventricular drain; $M C D=$ malformation of cortical development; $M E G$ = magnetoencephalography; $P V W M=$ periventricular white matter; TTE = time-to-event; VPS = ventriculoperitoneal shunt.

SUBMITTED June 27, 2019. ACCEPTED December 11, 2019

INCLUDE WHEN CITING Published online February 21, 2020; DOI: 10.3171/2019.12.PEDS19370. 
lowing hemispherectomy, and whether it should be considered a contraindication to surgery, remains a matter of debate. $1,3,5,12,19,24,27$ Recent reports have shown that seizure freedom can be obtained in patients with either bilateral MRI abnormalities ${ }^{12,19,26,28,34}$ or bilateral EEG abnormalities. ${ }^{21,34}$ However, other studies have shown that contralateral abnormalities on MRI may predict worse seizure outcome. . $^{3,27,34}$

We sought to evaluate the efficacy of hemispherectomy and determine whether contralateral MRI abnormalities can predict seizure recurrence.

\section{Methods \\ Study Design}

We performed a single-center retrospective observational cohort study of consecutive hemispherectomies performed at Miami Children's Hospital between January 2000 and June 2014. Miami Children's Hospital is a regional and international referral center for pediatric epilepsy. This is a high-volume academic center for epilepsy surgery currently staffed by 5 epileptologists, 3 neurosurgeons, and 4 neuroradiologists. We reviewed a prospectively maintained web-based database by an experienced senior epileptologist (I.M.). This database is updated in real time during epilepsy surgery conference or immediately following surgery.

\section{Patient Selection and Preoperative Evaluation}

Hemispherectomy was considered for pediatric patients ( $<19$ years old) with severe, refractory epilepsy related to unilateral hemispheric lesions. Hemispherectomy was also considered for bilateral but asymmetrical MRI lesions and seizures originating predominantly on one side. ${ }^{6}$ The decision to proceed with palliative hemispherectomy in these cases was influenced by the more extensive lesion on the ipsilateral side, the severity of the refractory epilepsy, the presence of side-appropriate hemiparesis and focal clinical features during seizures, and concordant electrophysiological data.

The preoperative investigations for hemispherectomy were tailored to each patient. $7,8,15,16,23,25$ All patients had a detailed medical history and physical examination. The history and medical records from the referral source were reviewed for consistency in the laterality of seizure semiology and EEG findings over the duration of the child's epilepsy, with particular attention to data at the time of onset. All patients underwent video EEG to document the seizure semiology and seizure onset and 1.5-T MRI preoperatively. Other diagnostic studies performed in a minority of patients included SPECT, PET, functional MRI, and Wada tests. All cases were reviewed at a weekly multidisciplinary epilepsy conference before considering hemispherectomy.

\section{MRI Technique and Classification}

MR images were acquired utilizing a 1.5-T magnet (GE Medical Systems)..$^{15}$ Our imaging protocol included T1weighted imaging, T2-weighted imaging, rapid gradient echo, and 3D volumetric acquisition spoiled gradient echo sequences. Closely spaced 3 -mm slices were utilized in all cases; special sequences including FLAIR have been routinely employed since 1996 at our institution, and surface coils are used to further define suspect areas. Scans were viewed in all 3 planes and results confirmed independently by 2 pediatric neuroradiologists; the first interpretation was blind to other findings and the second occurred at a multidisciplinary case conference after reviewing data from video EEG, ictal SPECT, and PET/interictal SPECT when available.

Information was collected on ipsilateral and contralateral MRI abnormalities, which were classified as cortical atrophy or signal change (gliosis), subcortical white matter $^{24}$ atrophy or signal change (gliosis), tuber, stroke, or malformation of cortical development (MCD).

\section{EEG Technique and Classification}

Video EEG monitoring and analysis was performed as previously reported. ${ }^{13,14}$ Preoperative ictal scalp EEG was prospectively recorded and dichotomized into seizures originating from the ipsilateral side of surgery versus nonlateralized onset. ${ }^{17}$

\section{Surgical Technique}

The primary surgical technique in this series and utilized at our institution was periinsular hemispherectomy, which was performed by all 3 neurosurgeons. This technique has been described in previous publications..$^{30-32}$ Anatomical hemispherectomy was used in instances when seizures were refractory to functional hemispherectomy. ${ }^{24}$

\section{Data Collection}

Data were collected from an institutional prospective epilepsy database in which all variables (clinical, radiological, EEG, outcome) are entered by treating neurologists in real time. We assessed outcomes using a time-to-event (TTE) Kaplan-Meier analysis., ${ }^{2,410}$ An event was defined as any seizures other than those in the first postoperative week, and auras. We also excluded unrelated seizures (e.g., following subsequent head injury, alcohol withdrawal) and those attributable to poor antiepileptic drug ${ }^{16}$ compliance or weaning of antiepileptic drugs. TTE analysis has several advantages over a yes/no seizure recurrence approach: 1) the analysis takes into account the changing seizure status and variability in duration of follow-up, therefore increasing our sample (patients with short follow-up can be included in this model); 2) the approach places a positive value in delay in seizure recurrence; 3) using Cox regression, it allows for examining the influence of various patient-level covariates on seizure outcome; and 4) it is statistically more powerful than the yes/no approach. However, the main disadvantage of this approach is that it will fail to account for the "running down phenomenon" (gradual decline of seizures over several months or years until seizure freedom is achieved following surgery). ${ }^{2,9} \mathrm{We}$ also collected data using the Engel classification of seizure outcomes, but this was not used for statistical analysis. ${ }^{33}$ Outcome data also included the presence and type of complications.

The variables extracted from the database include demographic data (patient sex, duration of epilepsy, age at time 
of surgery), seizure data (frequency, semiology, history of status epilepticus or ictal spams), side of surgery, history of presumed etiology, type of hemispherectomy (functional, anatomical), and pathological substrate. Radiological data include assessment of MRI, EEG, SPECT, PET, and source localization whenever available.

\section{Statistical Analysis}

We presented the raw data using descriptive statistics. For continuous variables, we presented means, standard deviations, and ranges. For categorical data, we presented frequencies and percentages. We created a Kaplan-Meier curve to demonstrate the overall time to seizure recurrence. We log-transformed nonnormally distributed quantitative variables. We removed any variable with less than 20 observations. We performed univariate Cox regression analysis to identify potential predictors of seizure outcome. We created Kaplan-Meier curves to graph the overall time-to-seizure recurrence function and for other categorical potential predictors of outcome. We compared the presence of contralateral abnormalities on MRI to the presence of contralateral ictal activity on scalp EEG using the Fisher exact test. Any variable with a $\mathrm{p}$ value $<0.05$ was considered significant. All statistics were performed in IBM SPSS Statistics (version 20, IBM Corp.).

\section{Results}

From January 2000 to June 2014, 69 patients (44 boys) with a mean $( \pm \mathrm{SD})$ age of $8.2 \pm 5.9$ years (range $0.1-20.8$ years) underwent 72 hemispherectomies at Miami Children's Hospital in Miami, Florida (Tables 1 and 2). Sixtyseven of these patients had a functional hemispherectomy, while another 5 had a completion of a previous functional hemispherectomy, including 2 completions of disconnection and 3 anatomical hemispherectomies. In addition, 16 patients underwent prior respective epilepsy surgery. Duration of epilepsy was $5.8 \pm 5.5$ years with 66 (91.7\%) having daily seizures. Ictal spasms were documented in 10 patients (13.9\%). Etiology included stroke in 28 (38.9\%), MCD in 11 (15.3\%), hemimegalencephaly in $11(15.3 \%)$, encephalitis in 13 (18.1\%), and other etiology in 7 patients (9.7\%). PET and SPECT scans were performed in a minority of patients undergoing hemispherectomy at our center and data were not available; thus, these variables were not included in the analysis. Magnetoencephalography (MEG) was not performed at our institution during the study period.

\section{Preoperative MRI and EEG}

Preoperative MRI was available in 69 patients. Of these 69 patients, contralateral MRI abnormalities were present in $21(30.4 \%)$. Of these, the main contralateral changes included periventricular white matter (PVWM) loss in 6 and PVWM gliosis in 5, migration disorder in 2, cortical tuber in 1 , stroke in 1 , cortical atrophy in 4 , and cortical signal abnormality (gliosis) in 2.

Of the 72 hemispherectomies performed, 10 (13.9\%) had contralateral ictal epileptic activity on EEG (nonlateralized) and $62(86.1 \%)$ had lateralized EEG data with no documented contralateral ictal activity. Of the 3 patients who underwent anatomical hemispherectomy, 2 did not
TABLE 1. Summary data (frequencies and percentages) for categorical preoperative variables

\begin{tabular}{|c|c|c|}
\hline Independent Variable & No. $(\%)^{*}$ & $\mathrm{p}$ Value \\
\hline \multicolumn{3}{|l|}{ Demographics } \\
\hline Males & $44(61.1)$ & \\
\hline Lt handedness & $22(30.6)$ & \\
\hline \multicolumn{3}{|l|}{ Seizure frequency } \\
\hline Daily & $66(91.7)$ & \\
\hline Weekly & $5(6.9)$ & \\
\hline Monthly & $1(1.4)$ & \\
\hline \multicolumn{3}{|l|}{ Seizure semiology } \\
\hline Primary generalized & $11(15.3)$ & \\
\hline Secondary generalized & $24(33.3)$ & \\
\hline \multicolumn{3}{|l|}{ History of status epilepticus } \\
\hline Partial seizures & $69(95.8)$ & \\
\hline Simple & $6(8.3)$ & \\
\hline Complex & $47(65.3)$ & \\
\hline Both & $11(15.3)$ & \\
\hline Infantile spasms & $10(13.9)$ & \\
\hline Prior surgery & $20(27.8)$ & \\
\hline \multicolumn{3}{|l|}{ Neurocognitive status } \\
\hline Normal & $13(18.1)$ & \\
\hline Developmental delay & $2(2.8)$ & \\
\hline Mental retardation & $13(18.1)$ & \\
\hline Learning disability & $31(43.1)$ & \\
\hline \multicolumn{3}{|l|}{ Etiology } \\
\hline MCD & $11(15.3)$ & \\
\hline Hemimegalencephaly & $11(15.3)$ & \\
\hline Rasmussen's encephalitis & $13(18.1)$ & \\
\hline Stroke & $28(38.9)$ & \\
\hline Other & $7(9.7)$ & \\
\hline \multicolumn{3}{|l|}{ Preop investigation } \\
\hline Contralateral MRI abnormalities & $21(30.4)$ & $0.009 \dagger$ \\
\hline PVWM loss & $6(8.7)$ & \\
\hline PVWM gliosis & $5(7.3)$ & \\
\hline Migrational disorder & $2(2.9)$ & \\
\hline Cortical tuber & $1(1.5)$ & \\
\hline Stroke & $1(1.5)$ & \\
\hline Cortical atrophy & $4(5.8)$ & \\
\hline Cortical gliosis & $2(2.9)$ & \\
\hline Contralateral ictal activity on EEG & $10(13.9)$ & $0.002 \dagger$ \\
\hline \multicolumn{3}{|l|}{ Hemispherectomy } \\
\hline Functional & $67(93.1)$ & \\
\hline Anatomical & $4(5.6)$ & \\
\hline Completion functional & $1(1.4)$ & \\
\hline Hemispherectomy, It-sided & $35(48.6)$ & \\
\hline
\end{tabular}

* Percentages are based on 69 patients or 72 hemispherectomies.

† Statistically significant using univariate Cox regression analysis. 
TABLE 2. Summary data (median, IQR, and range) for continuous preoperative variables

\begin{tabular}{lccl}
\hline \multicolumn{1}{c}{ Independent Variable } & $\begin{array}{c}\text { No. of } \\
\text { Cases }\end{array}$ & Median (IQR) & Range \\
\hline Age at first seizure, mos & 72 & $1.0(0.2-3.75)$ & $0-120$ \\
\hline Age at surgery, mos & 72 & $7.7(2.95-11.94)$ & $6-3000$ \\
\hline Duration of epilepsy, mos & 72 & $4.2(1.4-8.69)$ & $3-216$ \\
\hline
\end{tabular}

have contralateral EEG or MRI findings, whereas 1 had contralateral MRI findings but no data on EEG. Of the 2 patients who underwent completions of disconnection, 1 had contralateral EEG and MRI abnormalities, whereas the other did not have any contralateral EEG or MRI findings. Postoperative scans were performed in all patients. Imaging was available in all but 3 patients. The information (presence of contralateral imaging abnormality) was collected prospectively and entered into an institutional database. Completeness of disconnection was not analyzed in this study, and this is a limitation as this covariate is a predictor of seizure recurrence.

\section{Surgical Procedure}

Patients underwent periinsular hemispherotomy in 69 cases and anatomical hemispherectomy in 3 cases. Although the goal was seizure freedom in most cases, a significant proportion of our patients had putative negative risk factors, including $30.4 \%$ with contralateral MRI and $13.9 \%$ with contralateral ictal EEG abnormalities. In these cases, hemispherectomy was still offered with the goal of reducing seizure burden after extensive family counseling. The radiographic abnormalities mostly consist of minor periventricular signal changes and white matter loss or gliosis. However, in a smaller subset of patients, there was radiographic evidence of more extensive contralateral injuries and malformations, including cortical atrophy and polymicrogyria. In general, patients with subtle contra-

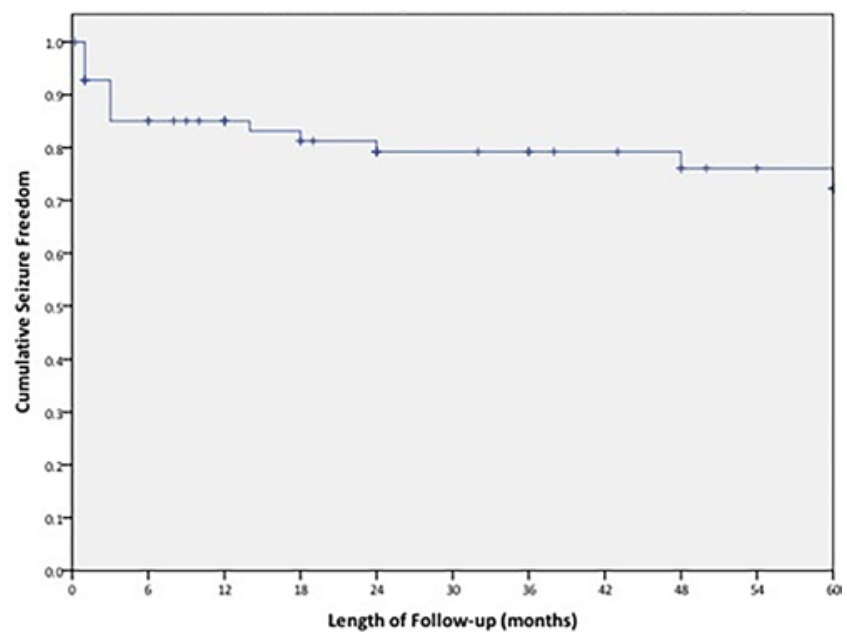

FIG. 1. Graph of seizure freedom function in patients with hemispherectomy. Figure is available in color online only.

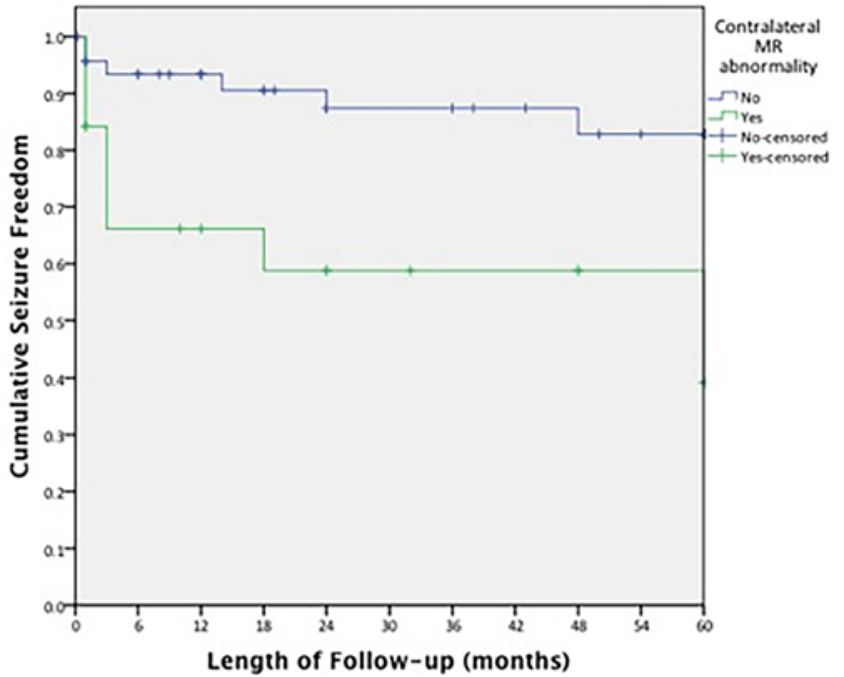

FIG. 2. Graph of seizure freedom function in patients with hemispherectomy according to contralateral MRI abnormality. Figure is available in color online only.

lateral findings underwent hemispherectomy with a curative goal. However, in the subset of children with obvious contralateral abnormalities, hemispherectomy was offered as a palliative approach, and parents were counseled accordingly. As an illustrative example, 2 infants with hemimegalencephaly were found to also have contralateral polymicrogyria. This was appreciated preoperatively and, following extensive discussion, the parents were offered hemispherectomy with a palliative goal, due to the frequency of preoperative seizures.

\section{Outcome}

Engel class I outcome was achieved in $59(86 \%)$ and $56(81 \%)$ patients at 1 and 2 years of follow-up, respectively. The mean time to seizure recurrence was $33.5 \pm$ 31.1 months (Fig. 1).

In univariate analyses, the absence of contralateral abnormalities on MRI (HR 4.09, 95\% CI 1.41-11.89, $\mathrm{p}=$ 0.009 ) was associated with a longer duration of seizure freedom (Fig. 2). When compared to the presence of contralateral ictal activity on video EEG using the Fisher exact test, we found that contralateral MRI abnormalities were significantly associated with nonlateralized EEG $(p=0.002)$. The absence of contralateral ictal activity on video EEG (HR 5.88, 95\% CI 1.96-17.65, $\mathrm{p}=0.002$ ) on univariate analysis was associated with longer duration of seizure freedom. The study was underpowered to determine if the efficacy of hemispherectomy is variable depending on the underlying etiology.

\section{Complications}

In this consecutive patient series, 15 patients experienced 20 complications $(20 / 72,27.8 \%)$, including the development of hydrocephalus necessitating CSF diversion in 9 cases (13\%), hygroma in 1, hemispheric edema in 1 , aseptic meningitis in 2, postoperative hemorrhage in 2, infection in 2, ischemic stroke in 2, and blood transfusion- 
contracted hepatitis $\mathrm{C}$ in 1 case. The incidence of shunted hydrocephalus in the overall cohort was $13 \%$, which decreased throughout the study period and was lower in the subgroup that received an external ventricular drain (EVD) after surgery. Additionally, there were a few cases of minor complications, including aseptic meningitis in 2 and development of hygroma in 1. Major, unexpected complications were rare. A 6-year-old boy with Rasmussen's encephalitis underwent left hemispherectomy. Postoperatively, MRI showed extensive edema in the disconnected frontal cerebral tissue obstructing the bilateral foramen of Monro, and enlargement of the contralateral ventricle despite an EVD in the surgical cavity. He was returned to the operating room for resection of the necrotic and overhanging frontal central white matter and intraventricular blood clot. The patient developed hydrocephalus requiring a ventriculoperitoneal shunt (VPS). A 3.5-year-old girl with septo-optic dysplasia and left hemispherectomy later developed a large epidural hematoma and hydrocephalus. She returned to the operating room 2 days later for hematoma evacuation. Two years after her hemispherectomy, she underwent an elective VPS insertion due to increasing ventriculomegaly. A 7-year-old girl with hypoxic/ischemic encephalopathy and enzymatic disorder underwent left hemispherectomy. Postoperatively, she developed thalamic stroke and hypothalamic dysfunction. She developed hydrocephalus shortly thereafter, requiring a VPS. A 7-year-old girl underwent right hemispherectomy and later developed a stroke of the left supplementary motor area causing transient facial weakness and persistent paresis, and she remains wheelchair dependent. A 6-yearold boy with type 1 neurofibromatosis and focal cortical dysplasia, who underwent a left hemispherectomy as a reoperation (following a left temporal lobectomy 2 years prior), developed a Staphylococcus surgical site infection 2 weeks postoperatively, requiring washout and removal of the bone flap. His bone was eventually replaced; he also developed hydrocephalus requiring a VPS. There were no deaths in this series.

\section{Discussion}

In this single-center retrospective cohort study, we found that bilateral MRI and EEG abnormalities were both associated with earlier seizure recurrence following hemispherectomy. The overall efficacy and complication profile in our series is similar to multiple previous singlecenter retrospective studies assessing outcome following hemispherectomy in children. ${ }^{12,16,18,19,22,24,26,27}$ Our patient population was generally similar to previous large retrospective studies in terms of patient demographics, etiology, and seizure semiology. 18,19,21,22

\section{What Is the Rate of Contralateral MRI Abnormalities?}

The rate of contralateral MRI abnormalities in our series was $30.4 \%$, which is comparable to previous reports $(26 \%-33 \%)$ that have used similar radiological criteria. ${ }^{3,22}$ Although the rate of contralateral MRI abnormalities in children undergoing hemispherectomy was almost three times higher (74\%) in one study, this was likely due to the inclusion of more subtle radiological changes (e.g., re- duced contralateral hemispheric volume) and patient selection bias. ${ }^{12}$

\section{Do Contralateral MRI Abnormalities Predict Outcome?}

The finding that bilateral MRI abnormalities are associated with a worse seizure outcome in patients undergoing hemispherectomy has been demonstrated by multiple other studies. , $^{3,54,27,34}$ In a recent study of 43 patients undergoing hemispherectomy, the 11 patients (26\%) who had significant contralateral MRI abnormalities had about half the seizure freedom rate of those with normal contralateral MRI. ${ }^{3}$ Bilateral abnormalities on preoperative MRI were also associated with worse cognitive outcome and neurocognitive development. ${ }^{3}$

However, some recent reports have shown that bilateral MRI abnormalities do not necessarily predict poor seizure outcome after hemsipherectomy. ${ }^{12,19,22,28}$ A retrospective study of 110 children undergoing hemispherectomy found contralateral MRI abnormalities in 74\%, with no impact on overall seizure outcome. ${ }^{12}$ However, contralateral MRI findings were only mild to moderate in most $(85 \%)$ of these patients and were always less prominent than the operated hemisphere. This study included subjective (nonvolumetric) assessments of reduction in contralateral hemisphere volume and sulcal change, which are known to be very subtle, even in diseases like hemimegalencephaly, raising questions as to its accuracy and/or clinical sgnificance. ${ }^{3,26}$ Also, when specifically evaluating for MCD and poststroke etiology subgroups in this study, the presence of contralateral abnormal cortical signal and thickness, respectively, were associated with a worse seizure outcome. ${ }^{12}$

Furthermore, a prior study from the same center in 50 patients with diffuse EEG findings, of whom 32 underwent hemispherectomy, found that bilateral MRI abnormalities almost halved the rate of seizure freedom, ${ }^{34}$ which is similar to our study and prior reports. ${ }^{3}$ However, in a recent follow-up study from the same group of 170 patients undergoing hemispherectomy, 5-year seizure freedom was unaffected by the presence of contralateral MRI abnormalities in the 56 patients (32.9\%) with these changes, irrespective of involvement of the cortex or not. ${ }^{22}$

These findings suggest that overt contralateral MRI findings may predict a worse seizure outcome in children undergoing hemispherectomy, especially when they involve the cortex..$^{3,22,34}$ However, data across different studies and different centers are contradictory, with some reporting good outcome despite bilateral findings. ${ }^{3,22}$ The TTE analysis utilized in our study is a more sensitive detector of "unfavorable" seizure outcome than studies assessing seizure outcome using the Engel classification at 1 year or final follow-up, which may include patients having nondisabling seizures in the favorable ("seizure-free") outcome group and may not account for eventual seizure recurrence in patients who are initially seizure free.

The patients with contralateral MRI abnormalities were more likely to harbor contralateral ictal seizures on noninvasive video EEG workup. The presence of contralateral ictal activity on EEG also predicted worse seizure outcome in our study, suggesting that the contralateral MRI changes may have been epileptogenic tissue, 
as both these variables were statistically associated with one another. However, multiple studies from different centers have shown that patients with bilateral ictal and interictal EEG findings do not necessarily go on to have a worse seizure outcome following hemispherectomy in the setting of a medically refractory hemispheric epileptic syndrome. ${ }^{3,21,34}$ This is likely related to the presurgical selection of patients in whom clinical features and imaging studies strongly suggest seizure origin predominantly or exclusively from the surgical hemisphere. ${ }^{22}$ Recent studies have suggested that other radiological modalities, such as $\mathrm{PET}^{22}$ and MEG, ${ }^{29}$ can help predict seizure outcome.

\section{Study Strengths}

The strengths of this study are as follows: 1) the analysis of outcomes was utilized, enhancing the statistical power to identify patient-level covariates that may predict seizure outcomes; 2) this is a relatively large series of consecutive pediatric patients with medically intractable epilepsy undergoing hemispherectomy; and 3) although the study was performed retrospectively, the data were collected prospectively and entered into a database by an experienced epileptologist.

\section{Study Limitations}

There are several limitations to this single-center retrospective study. It is a single-center study with an inherent patient selection bias, and hemispherectomies were performed by 3 surgeons, limiting generalizability of the results to other centers. For example, the high rate $(15 \%)$ of hemimegalencephaly is related to the referral basis to our institution and the patient population may not be representative of other centers, limiting the generalizability of our findings. The accuracy of results is limited by the accuracy of data input. The presurgical scalp EEG data available were limited to ictal activity, and background activity and interictal activity were not documented or analyzed, and these could represent unaccounted confounding variables. Whether contralateral interictal epileptiform discharges affect outcome was not examined as part of this study, although others have found excellent seizure outcome in patients undergoing hemispherectomy for well-selected candidates with generalized ictal or interictal activity. ${ }^{34}$ Although surgical technique was generally homogeneous across surgeons and throughout the study period, information regarding small variations in surgical technique (e.g., extent of temporal lobectomy, insulectomy, etc.) was not available in the prospective data registry that was used for data collection in this study and is thus not included in the analysis. Although no study has found a difference in outcome related to hemispherectomy techniques, small variations (e.g., insulectomy) have been related to outcome. ${ }^{11}$ The radiological assessment was not blinded to the patient; however, it was entered prospectively and as such was blinded to seizure outcome. Furthermore, although the presence of contralateral MRI abnormality was assessed, the completeness of disconnection-a wellknown predictor of seizure recurrence-was not assessed in the current study. Although seizure outcome is the most readily available marker of favorable outcome, we did not assess cognitive outcome or health-related quality of life, which may better reflect the desired outcome in children undergoing hemispherectomy. ${ }^{3}$ Overall, the limitations of this single-center study regarding the value of MRI in hemispherectomy are addressed in a multicenter follow-up study (Hemispherectomy Prediction Outcome Scale [HOPS] study) performed by the authors. Finally, although the complication profile and rate were not the focus of this paper, the complication rate in our series may reflect a referral basis of complex, difficult cases to our tertiary epilepsy center. The mortality rate and complication rate compare favorably to those in the literature. For example, although the shunt rate in the literature is highly variable across studies, the shunt rate of $13 \%$ in this cohort compares favorably to the reported rates in the literature, which range from $9 \%$ to $81 \% .{ }^{20}$ For example, in a recent multicenter study of 690 patients, the posthemispherectomy CSF shunt rate was $23 \%$, including $20 \%$ for functional and $30 \%$ for anatomical hemispherectomy. ${ }^{20}$

\section{Study Implications}

Children with severe, refractory epilepsy related to hemispheric syndrome associated with bilateral MRI abnormalities may have a higher likelihood of early seizure recurrence, and such preoperative findings should be considered a relative contraindication to "curative" hemispherectomy. However, the overall success rate in our study was favorable, with class I outcome achieved in $86 \%$ and $82 \%$ of patients at 1 and 2 years of follow-up, respectively. Although patients with contralateral MRI abnormalities may have earlier seizure recurrence, many patients still eventually have favorable seizure outcome. Alternatively, these patients may be considered candidates for "palliative" hemispherectomy, especially when noninvasive presurgical workup shows the concordant seizure onset zone originating predominantly from the diseased hemisphere. This should be taken into consideration during presurgical evaluation and family counseling.

\section{Conclusions}

Patients undergoing hemispherectomy with bihemispheric abnormalities may have a higher risk of earlier seizure recurrence, as evidenced by contralateral MRI abnormalities.

\section{Acknowledgments}

The authors wish to acknowledge the contributions of Dr. Sanjiv Bhatia, who passed away in May 2018. Dr. Bhatia conceived of this project and participated in review of the data as well as in the initial manuscript preparation. He was a role model and an inspiration to his colleagues and a miracle worker to his patients and their families. His professional legacy lives on in the children whose lives he improved by alleviating the burden of their seizures and in the success of the neurosurgical residents and fellows he trained.

\section{References}

1. Alexopoulos A, Lachhwani DK, Gupta A, Kotagal P, Harrison AM, Bingaman W, et al: Resective surgery to treat refractory status epilepticus in children with focal epileptogenesis. Neurology 64:567-570, 2005 
2. Berkovic SF, McIntosh AM, Kalnins RM, Jackson GD, Fabinyi GC, Brazenor GA, et al: Preoperative MRI predicts outcome of temporal lobectomy: an actuarial analysis. Neurology 45:1358-1363, 1995

3. Boshuisen K, van Schooneveld MMJ, Leijten FSS, de Kort GAP, van Rijen PC, Gosselaar PH, et al: Contralateral MRI abnormalities affect seizure and cognitive outcome after hemispherectomy. Neurology 75:1623-1630, 2010

4. Burneo JG, Villanueva V, Knowlton RC, Faught RE, Kuzniecky RI: Kaplan-Meier analysis on seizure outcome after epilepsy surgery: do gender and race influence it? Seizure 17:314-319, 2008

5. Chapman K, Wyllie E, Najm I, Ruggieri P, Bingaman W, Lüders J, et al: Seizure outcome after epilepsy surgery in patients with normal preoperative MRI. J Neurol Neurosurg Psychiatry 76:710-713, 2005

6. Ciliberto MA, Limbrick D, Powers A, Titus JB, Munro R, Smyth MD: Palliative hemispherotomy in children with bilateral seizure onset. J Neurosurg Pediatr 9:381-388, 2012

7. Duchowny M, Cross JH: Preoperative evaluation in children for epilepsy surgery, in Stefan H, Theodore WH (eds): Handbook of Clinical Neurology. Amsterdam: Elsevier, 2012, Vol 108, pp 829-839

8. Duchowny M, Jayakar P, Resnick T, Harvey AS, Alvarez L, Dean P, et al: Epilepsy surgery in the first three years of life. Epilepsia 39:737-743, 1998

9. Fallah A: Time to rethink how to measure postoperative seizure outcomes for potentially curative resective epilepsy surgery. Epilepsy Behav 41:53-54, 2014

10. Foldvary N, Nashold B, Mascha E, Thompson EA, Lee N, McNamara JO, et al: Seizure outcome after temporal lobectomy for temporal lobe epilepsy: a Kaplan-Meier survival analysis. Neurology 54:630-634, 2000

11. Griessenauer CJ, Salam S, Hendrix P, Patel DM, Tubbs RS, Blount JP, et al: Hemispherectomy for treatment of refractory epilepsy in the pediatric age group: a systematic review. $\mathbf{J}$ Neurosurg Pediatr 15:34-44, 2015

12. Hallbook T, Ruggieri P, Adina C, Lachhwani DK, Gupta A, Kotagal P, et al: Contralateral MRI abnormalities in candidates for hemispherectomy for refractory epilepsy. Epilepsia 51:556-563, 2010

13. Jayakar P, Duchowny M, Resnick TJ, Alvarez LA: Localization of seizure foci: pitfalls and caveats. J Clin Neurophysiol 8:414-431, 1991

14. Jayakar P, Duchowny MS, Resnick TJ, Alvarez LA: Localization of epileptogenic foci using a simple reference-subtraction montage to document small interchannel time differences. J Clin Neurophysiol 8:212-215, 1991

15. Jayakar P, Dunoyer C, Dean P, Ragheb J, Resnick T, Morrison $\mathrm{G}$, et al: Epilepsy surgery in patients with normal or nonfocal MRI scans: integrative strategies offer long-term seizure relief. Epilepsia 49:758-764, 2008

16. Jayakar P, Gaillard WD, Tripathi M, Libenson MH, Mathern GW, Cross JH: Diagnostic test utilization in evaluation for resective epilepsy surgery in children. Epilepsia 55:507-518, 2014

17. Johnston CE II, Happel LT Jr, Norris R Jr, Burke SW, King AG, Roberts JM: Delayed paraplegia complicating sublaminar segmental spinal instrumentation. J Bone Joint Surg Am 68:556-563, 1986

18. Jonas R, Nguyen S, Hu B, Asarnow RF, LoPresti C, Curtiss $\mathrm{S}$, et al: Cerebral hemispherectomy: hospital course, seizure, developmental, language, and motor outcomes. Neurology 62:1712-1721, 2004

19. Kossoff EH, Vining EPG, Pillas DJ, Pyzik PL, Avellino AM, Carson BS, et al: Hemispherectomy for intractable unihemispheric epilepsy etiology vs outcome. Neurology 61:887890,2003

20. Lew SM, Matthews AE, Hartman AL, Haranhalli N: Posthe- mispherectomy hydrocephalus: results of a comprehensive, multiinstitutional review. Epilepsia 54:383-389, 2013

21. Limbrick DD, Narayan P, Powers AK, Ojemann JG, Park TS, Bertrand M, et al: Hemispherotomy: efficacy and analysis of seizure recurrence. J Neurosurg Pediatr 4:323-332, 2009

22. Moosa ANV, Gupta A, Jehi L, Marashly A, Cosmo G, Lachhwani D, et al: Longitudinal seizure outcome and prognostic predictors after hemispherectomy in 170 children. Neurology 80:253-260, 2013

23. Obeid M, Wyllie E, Rahi AC, Mikati MA: Approach to pediatric epilepsy surgery: State of the art, part I: general principles and presurgical workup. Eur J Paediatr Neurol 13:102-114, 2009

24. Peacock WJ, Wehby-Grant MC, Shields WD, Shewmon DA, Chugani HT, Sankar R, et al: Hemispherectomy for intractable seizures in children: a report of 58 cases. Childs Nerv Syst 12:376-384, 1996

25. Perry MS, Duchowny M: Surgical management of intractable childhood epilepsy: curative and palliative procedures. Semin Pediatr Neurol 18:195-202, 2011

26. Salamon N, Andres M, Chute DJ, Nguyen ST, Chang JW, Huynh MN, et al: Contralateral hemimicrencephaly and clinical-pathological correlations in children with hemimegalencephaly. Brain 129:352-365, 2006

27. Smith SJM, Andermann F, Villemure JG, Rasmussen TB, Quesney LF: Functional hemispherectomy: EEG findings, spiking from isolated brain postoperatively, and prediction of outcome. Neurology 41:1790-1794, 1991

28. Terra-Bustamante VC, Inuzuka LM, Fernandes RMF, Escorsi-Rosset S, Wichert-Ana L, Alexandre V Jr, et al: Outcome of hemispheric surgeries for refractory epilepsy in pediatric patients. Childs Nerv Syst 23:321-326, 2007

29. Torres CV, Fallah A, Ibrahim GM, Cheshier S, Otsubo H, Ochi A, et al: The role of magnetoencephalography in children undergoing hemispherectomy. J Neurosurg Pediatr 8:575-583, 2011

30. Villemure JG: Anatomical to functional hemispherectomy from Krynauw to Rasmussen. Epilepsy Res Suppl 5:209215, 1992

31. Villemure JG, Mascott CR: Peri-insular hemispherotomy: surgical principles and anatomy. Neurosurgery 37:975-981, 1995

32. Villemure JG, Rasmussen T: Functional hemispherectomy in children. Neuropediatrics 24:53-55, 1993

33. Wieser HG, Blume WT, Fish D, Goldensohn E, Hufnagel A, King D, et al: ILAE Commission Report. Proposal for a new classification of outcome with respect to epileptic seizures following epilepsy surgery. Epilepsia 42:282-286, 2001

34. Wyllie E, Lachhwani DK, Gupta A, Chirla A, Cosmo G, Worley S, et al: Successful surgery for epilepsy due to early brain lesions despite generalized EEG findings. Neurology 69:389-397, 2007

\section{Disclosures}

The authors report no conflict of interest concerning the materials or methods used in this study or the findings specified in this paper.

\section{Author Contributions}

Conception and design: Ragheb, Weil, Fallah, Ibrahim, Bhatia. Acquisition of data: Weil, Wang. Drafting the article: Weil. Critically revising the article: Fallah, Ibrahim, Elkaim, Jayakar, Miller, Niazi. Reviewed submitted version of manuscript: Weil, Fallah, Wang, Ibrahim, Elkaim, Jayakar, Miller, Niazi.

\section{Correspondence}

John Ragheb: Nicklaus Children's Hospital, Miami, FL. john. ragheb2@nicklaushealth.org. 